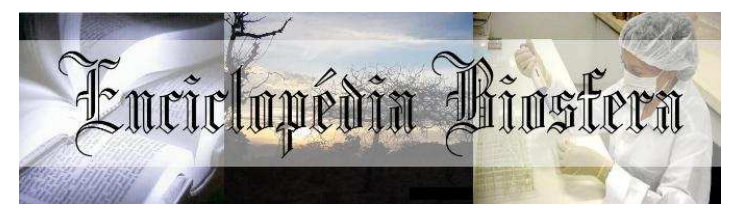

\title{
DESCRIÇÃO DO APOIO SOCIAL OFERTADO ÀS MULHERES EM TRATAMENTO DO CÂNCER DE MAMA: IMPORTÂNCIA PARA O PROGNÓSTICO
}

Sabrina de Almeida Rodrigues ${ }^{1}$; Julia Wosch Brochonski ${ }^{1}$; Felippe Fernandes ${ }^{1}$; Marcelo Picinin Bernuci².

Acadêmicos do Curso de Medicina do Centro Universitário Cesumar -

UNICESUMAR, Maringá - Paraná; sabrinaarodrigues@hotmail.com;

2Orientador e docente do programa de Pós Graduação em promoção da Saúde do Centro Universitário Cesumar - UNICESUMAR, Maringá - Paraná e associado ao Instituto Cesumar de Ciência, Tecnologia e Inovação (ICETI).

Recebido em: 02/10/2017 - Aprovado em: 21/11/2017 - Publicado em: 05/12/2017 DOI: 10.18677/EnciBio 2017B95

\begin{abstract}
RESUMO
A adesão ao tratamento de câncer de mama está associada à maior sobrevida, porém, fatores associados ao abandono não são amplamente compreendidos. As variáveis psicossociais têm recebido uma atenção limitada, apesar das relações bem documentadas com a adesão aos tratamentos de outras doenças crônicas. $O$ Objetivo deste foi descrever o perfil do apoio social ofertado às pacientes em tratamento do câncer de mama bem como sua relação com o prognóstico.Tratou-se de um estudo descritivo realizado em um centro de referência na investigação de câncer de mama do município de Maringá/Paraná a partir da análise de prontuários de mulheres diagnosticadas com câncer de mama do tipo maligno(CID C50) entre os anos de 2013 e 2014. Os dados psicossociais foram obtidos por meio da aplicação de um questionário semiestruturado durante visita domiciliar. Foram identificadas no período 98 mulheres com diagnóstico de câncer de mama. Das residentes em Maringá, $56 \%(\mathrm{n}=28)$ aceitaram participar da pesquisa. Das entrevistadas, $28,6 \%$ relataram ter vivenciado algum episódio de depressão ou transtorno psicológico durante o período de tratamento e $35,7 \%$ passaram por um período de estresse e dificuldade. $82 \%$ relataram ter tido apoio social durante 0 tratamento, das quais $47,8 \%$ eram casadas; $26,1 \%$ viúvas; $17,4 \%$ separadas e $8,7 \%$ solteiras.Das pacientes que receberam suporte social, 39\% obtiveram alta no tratamento, 43,5\% ainda estavam em tratamento e 17,5\% apresentaram recidivas. Das pacientes que não receberam apoio, $20 \%$ obtiveram alta e $80 \%$ estavam em tratamento. O apoio social oferecido às mulheres sob tratamento de câncer de mama influencia positivamente o prognóstico.
\end{abstract}

PALAVRAS-CHAVE: Apoio social. Câncer de mama. Doença crônica. 


\title{
DESCRIPTION OF THE SOCIAL SUPPORT OFFERED TO WOMEN IN TREATMENT OF BREAST CANCER: IMPORTANCE FOR PROGNOSIS
}

\begin{abstract}
The adherence to the treatment of breast cancer is associated with higher survival, but the factors associated with abandonment are not widely understood. Psychosocial variables have received limited attention despite their well-documented connections with the adherence to other chronic diseases. To describe the profile of the social support offered to patients that are in breast cancer treatment, as well as its relation with the prognosis. This is a descriptive study carried out in a reference center in breast cancer research in the municipality of Maringá/Paraná, based on the analysis of medical records of women diagnosed with malignant breast cancer (CID C50) between the years of 2013 and 2014. Psychosocial data were obtained through the application of asemi-structured questionnaire during home visit. A total of 98 women diagnosed with breast cancer were identified. Of the residents in Maringá, $56 \%(n=28)$ accepted to participate in the research. $28.6 \%$ of the interviewees reported experiencing any episode of depression or psychological distress during the treatment period and $35.7 \%$ went through a period of stress and difficulties. $82 \%$ reported having had social support during treatment, of which $47.8 \%$ were married; $26.1 \%$ widows; $17.4 \%$ separated and $8.7 \%$ singles. Of the patients who received social support, 39\% were discharged from treatment, $43.5 \%$ were still receiving treatment, and $17.5 \%$ presented relapses. Of the patients who did not receive support, $20 \%$ of them were discharged and $80 \%$ were undergoing treatment. The social support offered to women under breast cancer treatment positively influences the prognosis.
\end{abstract}

KEYWORDS: Chronic disease; Breast cancer; Social support.

\section{INTRODUÇÃO}

A prevalência do câncer de mama é a maior, tanto mundial quanto nacionalmente, ao se tratar de tumores malignos em pessoas do sexo feminino (INCA, 2016). Esta malignidade corresponde a $28 \%$ da incidência anual de câncer (INCA, 2016). Estima-se que mais de 58 mil casos se manifestarão no período bienal $2016-2017$, ocupando $28,1 \%$ de todas as neoplasias malignas em mulheres (INCA, 2016). A incidência do câncer de mama no Brasil alargou-se de maneira significativa, isso deve-se tanto ao aumento da expectativa de vida inerente às melhores condições de vida quanto à grande campanha de rastreio de câncer de mama implementadas no país recentemente (INCA, 2016).

Em contrapartida, nota-se o aumento da mortalidade decorrente do câncer de mama, devido principalmente ao diagnóstico tardio da doença e a não adesão e abandono do tratamento proposto (INCA, 2015). Quando não tratado, o câncer de mama pode levar a consequências diversas como ulcerações cutâneas, comprometimento linfonodal, principalmente de cadeia axilar e subclávia, linfedema de membros superiores, metástases localizadas e disseminadas, e até ao óbito (RIIHIMAKI, 2012). Dessa forma, a manutenção e a persistência do tratamento são essenciais para um bom prognóstico.

Após a descoberta de um nódulo na mama, as dúvidas e incertezas são estabelecidas e podem ou não ser "atenuadas" através do exame físico e exames radiológicos (FURLANETTO, 2000). Quando há a confirmação de que é um tumor maligno, a mulher passará por várias fases de conflito interno que oscilam desde a negação da doença, em que a paciente (e familiares) procuram diversos 
profissionais na esperança de que algum lhe dê um diagnóstico contrário aos achados, até a fase final em que haverá a aceitação da existência do tumor (MALUF, 2004).

Mudanças de humor, padrão de vida e comportamento ligados a um quadro depressivo são comuns em portadoras do câncer de mama e tornam-se fatores agravantes no curso natural da doença interferindo diretamente no prognóstico, no entanto frequentemente são desprezadas. Essas alterações biopsicológicas pelas quais passa a paciente portadora da neoplasia geralmente estão abertamente associadas aos procedimentos que visam o controle da mesma tais como cirurgia, quimioterapia, radioterapia, a hormonioterapia $e$ até mesmo 0 próprio acompanhamento de controle após a resolução da patologia (FURLANETTO, 2000).

Ainda não está claro o motivo do abandono ao tratamento em qualquer uma das fases. Há estudos demonstrando que as principais razões pelo abandono ao tratamento são: progressão da doença, efeitos colaterais, como por exemplo, artralgias, eventos tromboembólicos, além de calafrios, sudorese noturna, efeitos gastrointestinais e morte (CHIRGWIN, 2016), outros evidenciam que a principal causa é a falta de comunicação e clareza por parte de profissionais de saúde quanto ao tratamento (SAWESI, 2014) e o suporte social pode estar intimamente relacionado à continuidade da terapia proposta para o câncer de mama (MAGAI, 2007).

Algumas evidências sugerem a presença de fatores emocionais e existenciais como influenciadores no processo de evolução da doença e na eficácia terapêutica (CHIRGWIN, 2016), esclarecendo a necessidade de compreender o impacto biopsicológico criado pelo câncer de mama e seus sintomas pertencentes a um quadro depressivo. As alterações do pensamento e do comportamento inerentes aos sintomas depressivos podem influenciar tanto somaticamente, agravando os sintomas físicos do câncer de mama quanto psicologicamente o que gera prejuízo funcional, menor adesão terapêutica, piora da qualidade de vida, pior prognóstico e consequentemente elevam as taxas de morbidade e mortalidade (SOUZA, 2013).

A depressão é evidenciada como a doença mais incapacitante em mulheres em todo o mundo (DE ANDRADE et al., 2006). Ainda é inconclusiva a associação entre depressão e mortalidade ao se tratar do câncer de mama (FALAGAS, 2007). Alguns autores observaram que a depressão influencia no aumento da mortalidade (GOODWIN, 2004). Porém, outros afirmam que estresses psicossociais não alteram o curso natural da doença nem da mortalidade (PHILLIPS, 2008).

Embora supostamente o suporte social seja um fator fundamental para adesão ao tratamento, estudos que comprovem a eficácia deste suporte ainda são escassos. Os objetivos do presente estudo foram verificar a prevalência de sintomas depressivos em mulheres com câncer de mama, analisar os fatores de risco associados aos quadros depressivos em mulheres com neoplasia e retratar o perfil do apoio social ofertado às pacientes em tratamento do câncer bem como sua influência no prognóstico.

\section{MATERIAL E MÉTODOS}

Tratou-se de um estudo descritivo do tipo transversal cuja população investigada foi de mulheres diagnosticadas com câncer de mama residentes no Município de Maringá, Paraná. O estudo foi realizado na Clínica da Mulher, serviço de referência para câncer de mama no Município no período compreendido entre os anos de 2013 e 2014. 
Os dados referentes à caracterização sóciodemográfica e fatores de risco para o câncer de mama foram coletados dos prontuários eletrônicos e aqueles referentes às questões psicossociais, que podem estar relacionadas e contribuem para a evolução da doença, coletados a partir da aplicação de um questionário semiestruturado durante visita domiciliar. As pacientes foram contatadas via telefone para agendamento da visita domiciliar. Apenas participaram do estudo as pacientes que assinaram o termo de consentimento livre e esclarecido (TCLE).

O número de mulheres diagnosticadas com neoplasia maligna de mama no período descrito totalizou 98 mulheres, das quais $49 \%$ residiam em outros municípios e foram encaminhadas para a Clínica da Mulher e $51 \%$ residiam no município de Maringá. Das residentes, 56\% ( $n=28)$ aceitaram responder ao questionário e foram selecionadas para participar da pesquisa. Destas, 32\% das residentes não atenderam após três ligações em dias e horários diferentes, 10\% recusaram-se a participar e $2 \%$ foi identificado como óbito. Portanto, a amostra final foi de 28 mulheres que aceitaram responder um questionário relacionado à neoplasia de mama, contendo dados sócio-demográficos, hábitos de vida, fatores de risco que podem estar associados ao câncer de mama. Este estudo foi aprovado pelo comitê de ética do Centro Universitário Cesumar CEP/Cesumar sob o parecer no 1.092 .279 em 03/06/2015.

\section{RESULTADOS E DISCUSSÃO}

Estudos realizados com mulheres direcionados a análise de fatores psicológicos nas pacientes portadoras de câncer de mama são escassos. Buscouse, portanto, no presente estudo descrever os fatores psicossociais que influenciaram a qualidade de vida das pacientes em tratamento de câncer de mama. O total de mulheres diagnosticadas com neoplasia maligna de mama no período descrito foi de 98 , das quais $49 \%$ residiam em outros municípios e foram encaminhadas para a Clínica da Mulher e 51\% residiam no município de Maringá. Das residentes $(n=50), 32 \%$ não atenderam após três ligações em dias e horários diferentes, $10 \%$ recusaram-se a participar e $2 \%$ haviam falecido no período. Assim $56 \%(n=28)$ das pacientes participaram do estudo.

Os dados referentes à relação entre estado civil das pacientes e a presença/ausência de apoio psicológico estão apresentados na Tabela 1. Notou-se se que a maioria das pacientes são casadas, das quais mais de $90 \%$ relataram ter recebido apoio social durante todo o processo de tratamento da doença. As solteiras, embora em minoria, receberam em $100 \%$ dos casos, apoio social de suas famílias e amigos. Diferentemente das pacientes viúvas e separadas, apenas $67 \%$ e $80 \%$, respectivamente, receberam apoio. Este fato pode se explicar pela desestruturação familiar que ocorre durante o processo de falecimento do conjugue ou separação. Ambos, ausência de apoio e o próprio processo de desestruturação familiar, podem contribuir para uma pior adaptação física e psicossocial da paciente ao tratamento do câncer de mama, uma vez que há evidências de que a manutenção de um relacionamento estável e de laços familiares pode beneficiar o prognóstico da paciente durante o longo protocolo de tratamento (CANAVARRO, 2015; WEI, 2016).

De modo geral, o câncer de mama tem como características na paciente, um aumento no senso de responsabilidade em relação a si mesma, uma maior vulnerabilidade, mudanças na auto-estima, raiva, medo da morte, de mudanças/da perda e mudanças na autoimagem, perda da feminilidade (WILMOTH, 1996). Outras mudanças corpóreas e não fisiológicas incluem, mal-estar geral (inclusive na 
possibilidade de diminuição da imunidade e infecções oportunistas), náuseas e vômitos importantes, diminuição da função cognitiva (como alterações na memória entre outras), quadro clínico decorrente de hipoestrogenismo por diminuição da função ovariana (principalmente em pacientes pré-menopausadas, podendo desenvolver diminuição da lubrificação vaginal, atrofia vaginal, fogachos, amenorréia) (HANSON FROST, 2000). Nesta fase, o apoio familiar e o suporte médico/psicológico pode amenizar os possíveis efeitos colaterais com medicações específicas e evoluções, diminuindo as incertezas da paciente (MELO, 2012).

TABELA 1: Caracterização do estado civil e apoio psicológico durante o tratamento.

\begin{tabular}{cccc}
\hline Estado civil & Com apoio & Sem apoio & Total \\
\hline Casada & $91,7 \%$ & $8,3 \%$ & 12 \\
Solteira & $100 \%$ & $0 \%$ & 2 \\
Viúva & $66,7 \%$ & $33,3 \%$ & 9 \\
Separada & $80 \%$ & $20 \%$ & 5 \\
\hline
\end{tabular}

A descrição da presença de fatores estressores ou episódios depressivos no período entre diagnóstico e tratamento da neoplasia estão demonstrados na Figura 1. Notou-se que no decorrer do tratamento as pacientes passaram por eventos geradores de estresse tais como: dor, imobilidade dos membros superiores, pobre percepção do estado de saúde e inclusive dificuldades financeiras (35\%) e episódios depressivos (26\%). Estes episódios quando manejados de forma efetiva seja por intervenção médica ou por suporte psicossocial das diversas instituições como família, igreja e grupos de apoio estão relacionados com uma melhor qualidade de vida das pacientes portadoras da doença (MANSANO-SCHLOSSER, 2016; SHAKERI, 2016), consequentemente otimizando as terapias adjuvantes no controle e tratamento do câncer de mama.

Ainda que recorrentes, os sintomas depressivos são muitas vezes subestimados nos serviços de saúde (VAHDANINIA, 2010). Indivíduos que desenvolvem um quadro depressivo durante o curso de uma patologia tendem a apresentar exacerbações de sintomas físicos, prejuízo funcional, menor adesão aos tratamentos propostos, diminuição dos comportamentos de autocuidado e piora da qualidade de vida e ainda pior prognóstico, com maiores morbidades e mortalidades (SOUZA, 2013).

Um grande número de estudos focados na variação psicológica compara pacientes mastectomizadas com outras submetidas a cirurgias conservadoras. Estes evidenciam que, quanto mais conservadora a cirurgia, menor o impacto psicológico causado pela perda da autoimagem. Porém, não há unanimidade quanto a isso, havendo outros estudos que afirmam não haver diferença entre o impacto psicológico em pacientes submetidas a mastectomia radical ou cirurgia conservadora (MALUF, 2004). De qualquer forma, mostrou-se nesse estudo que muitas mulheres em tratamento do câncer de mama relataram estar sob algum tipo de fator estressor, que não está necessariamente ligado à mastectomia, e deve, portanto, ser considerado durante todo o processo de tratamento. Os manifestos de terem vivenciado em algum momento episódios depressivos ressalta a importância de considerar questões psicossociais no manejo e na atenção destas pacientes. 


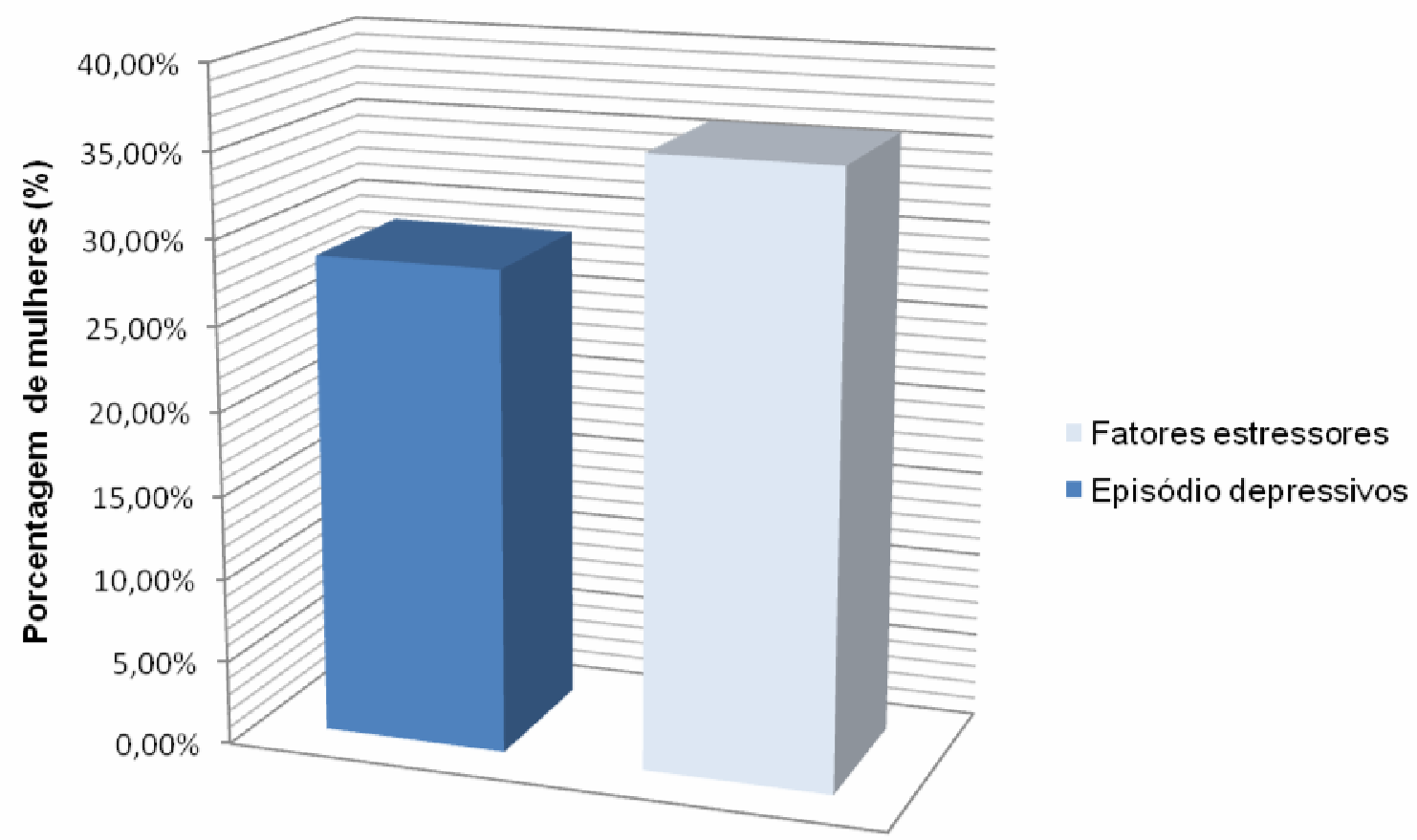

FIGURA 1 : Presença de fatores estressores ou episódios depressivos no período durante o período de tratamento da neoplasia.

Fonte: Elaborado pelos autores (2017)

Os dados referentes ao prognóstico das pacientes que receberão ou não apoio social durante o período de tratamento da neoplasia estão apresentados na Figura 2. Notou-se que as mulheres que receberam apoio social apresentaram maior controle da doença $(32,1 \%)$ contrastando com as que não receberam apoio, apresentando índices representativamente menores de controle da neoplasia $(3,6 \%)$. Notou-se que os índices de recidivas foram maiores naquelas em que 0 apoio se fez presente $(7,1 \%)$.

A reação ao diagnóstico de câncer de mama depende de fatores como a personalidade da paciente, aspectos da doença, do tratamento e do meio social no qual a paciente está inserida (MALUF, 2004). Existem evidências que associam a fase de suspeita da neoplasia com a propensão ao isolamento, ocorrendo como conseqüência, o atraso no diagnóstico e a má adesão ao tratamento (MENEZES, 2012). Sendo assim, é muito importante que a paciente tenha suporte de sua família e amigos durante todo esse processo. A neoplasia mamária influencia toda a família, sendo esta de extrema importância para o prognóstico da mulher, esse apoio estimula a paciente a defrontar-se com os desafios relacionados ao câncer de mama, ocorrendo uma melhor adequação no curso da doença (PRADO; DO, 2016). Por isso, faz-se necessário um suporte psicológico satisfatório e efetivo em todas as fases do tratamento para que isso reflita em maior resignação na conduta e qualidade de vida da mulher. Estudos também revelam que o suporte psicossocial faz-se importante ao tratar o medo das pacientes em relação às recidivas da doença, provocando impacto direto no progresso das terapias de suporte e tratamento (GANZ, 2008). 


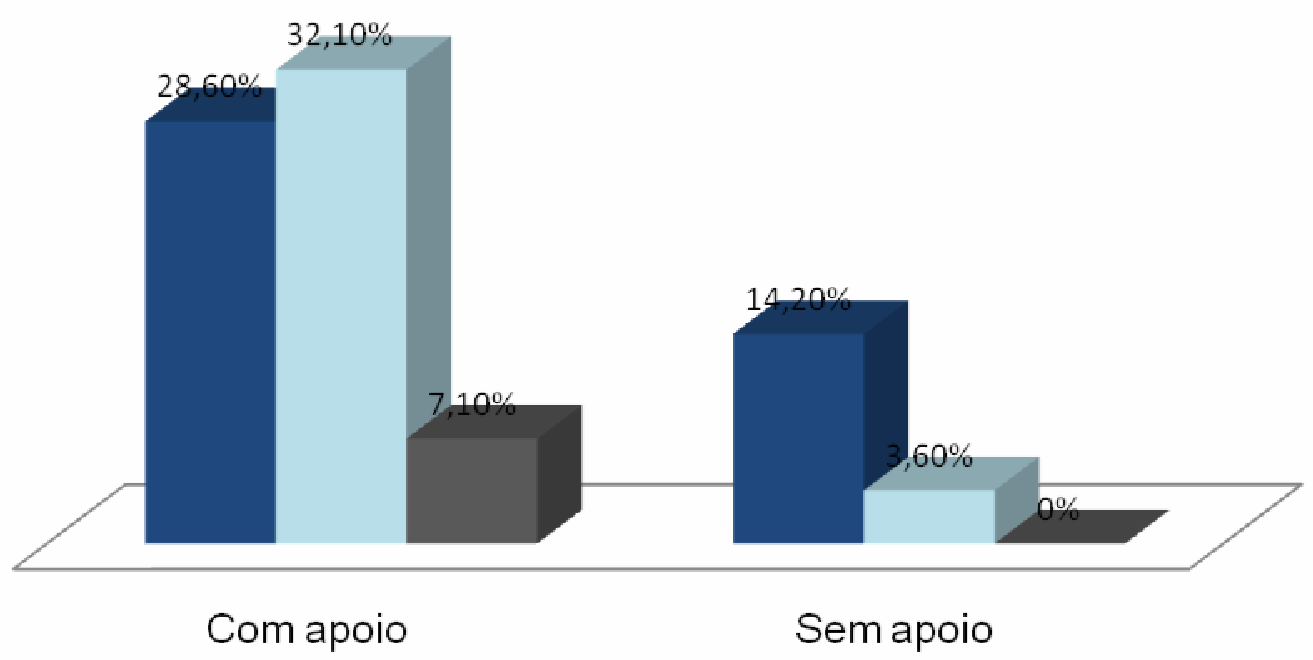

FIGURA 2: Prognóstico das pacientes que obtiveram ou não apoio social durante o período de tratamento da neoplasia.

Fonte: Elaborado pelos autores.

\section{CONCLUSÃO}

Este estudo demonstra que o apoio e boa relação com amigos e família auxiliam no enfrentamento a essa nova fase de vida proporcionada pelo diagnóstico na neoplasia e tratamento, favorecendo um ambiente mais leve para enfrentar a doença com maior adesão e autoconfiança, diminuindo os índices de depressão e angústias, ao mesmo tempo em que aumenta as chances de regressão e cura da doença. Além disso, poucos estudos recentes tratam do tema, refletindo uma maior necessidade de estudos nesse assunto que abordem o aspecto psicológico da paciente diagnosticada com a neoplasia mamária.

\section{REFERÊNCIAS}

CANAVARRO, M. C.; SILVA, S.; MOREIRA, H. Is the link between post traumatic grow than anxious symptoms mediated by marital intimacy in breast cancer patients?. European Journal of Oncology Nursing, v. 19, n. 6, p. 673-679, 2015. Disponível em: DOI:10.1016/j.ejon.2015.04.007> $<$ http://dx.doi.org/10.1016/j.ejon.2015.04.007

CHIRGWIN, J. H.; GIOBBIE-HURDER, A.; COATES, A.S.; PRICE, K. N.; EJLERTSEN, B.; DEBLED, M.; GELBER, R. D.; GOLDHIRSCH, A.; SMITH, I.; RABAGLIO, M.; FORBES, J. F.; NEVEN, P.; LÁNG, I.; COLLEONI, M. THÜRLIMANN, B. Treatment adherence and its impact on disease-free survival in the Breast International Group 1-98 trial of tamoxifen and letrozole, alone and in sequence.Journal of clinical oncology, v. 34, n. 21, p. 2452-2459, 2016 Disponível em: <http://ascopubs.org/doi/abs/10.1200/JCO.2015.63.8619> 
DE ANDRADE, L. H. S. G.; VIANA, M. C.; SILVEIRA, C. M.. Epidemiologia dos transtornos psiquiátricos na mulher. Revista de psiquiatria clínica. (São Paulo), p. 43-54, 2006. Disponível em: <http://dx.doi.org/10.1590/S0101-60832006000200003>

FALAGAS, M. E.; ZARKADOULIA, E. A.; IOANNIDOU, E. N.; PEPPAS, G.; CHRISTODOULOU, C.; RAFAILIDIS P. I. The effect of psychosocial factors on breast cancer out come: a systematic review. Breast Cancer Research, v. 9, n. 4, p. R44, 2007. Disponível em: <https://breast-cancerresearch.biomedcentral.com/articles/10.1186/bcr1744>

FURLANETTO, L. M.; CAVANAUGH S. V. A.; BUENO, J. R.; CREECH, S. D.; POWELL, L. H. Association between depressive symptoms and mortality in medical inpatients. Psychosomatics, v. 41, n. 5, p. 426-432, 2000. Disponível em: <http://dx.doi.org/10.1176/appi.psy.41.5.426>

GANZ, P. A. Psychological and social aspects of breast cancer. Oncology Journal, v. 22, n. 6, p. 642, 2008. Disponível em: <http://www.cancernetwork.com/oncologyjournal/psychological-and-social-aspects-breast-cancer>

GEBRIM, L. H.; QUADROS, L. G. A. Rastreamento do câncer de mama no Brasil. Revista Brasileira de Ginecologia e Obstetetrícia. 2006, vol.28, n.6, pp.319-323. ISSN 0100-7203. Disponível em: <http://dx.doi.org/10.1590/S010072032006000600001. >

GOODWIN, J, S.; ZHANG, D. D.; OSTIR, G. V. Effect of depression on diagnosis, treatment, and survival of older women with breast cancer. Journal of the American Geriatrics Society, v. 52, n. 1, p. 106-111, 2004. Disponível em: <http://onlinelibrary.wiley.com/doi/10.1111/j.1532-5415.2004.52018.x/abstract>

HANSON FROST, M.; SUMAN, V. J.; RUMMANS, T. A.; DOSE, A. M.; TAYLOR, M.; NOVOTNY, P.; JOHNSON, R.; EVANS, R. E. Physical, psychological and social well-being of women with breast cancer: the influence of disease phase. Psycho-Oncology, v. 9, n. 3, p. 221-231, 2000. Disponível em: <http://onlinelibrary.wiley.com/doi/10.1002/1099-1611(200005/06)9:3\%3C221::AIDPON456\%3E3.0.CO;2-T/full>

INCA - Instituto Nacional de Câncer José Alencar Gomes da Silva . Câncer tipo Mama. Rio de Janeiro: INCA; 2016. Disponível em: $<$ http://www2.inca.gov.br/wps/wcm/connect/tiposdecancer/site/home/mama/cancer_ mama+>

MAGAI, C.; CONSEDINE, N.; NEUGUT, A. I.; HERSHMAN, D. L. Common psychosocial factors under lying breast cancer screening and breast cancer treatment adherence: a conceptual review and synthesis. Journal of Women's Health, v. 16, n. 1, p. 11-23, 2007. Disponível em: <http://online.liebertpub.com/doi/abs/10.1089/jwh.2006.0024>

MALUF, M. F. M. A sexualidade das pacientes submetidas à mastectomia radical [monografia]. São Paulo: Universidade de São Paulo, 2004. Disponível em: $<$ www.teses.usp.br/teses/disponiveis/5/5139/tde.../MariaFernandadeMatosMaluf.pdf 
MANSANO-SCHLOSSER, T. C.; CEOLIM, M. F.; VALERIO, T. D. Poor sleep quality, depression and hope before breast cancer surgery. Applied Nursing Research, v. 34, p. 7-11, 2017. Disponível em: <http://dx.doi.org/10.1016/j.apnr.2016.11.010>

MELO, M. C. B. DE; BARROS, E. N.; CAMPANELLO, M. C. V. A.; FERREIRA, L. Q. L.; ROCHA, L. L. C.; SILVA, C. I. M. G.; SANTOS, N. T. F. O funcionamento familiar do paciente com câncer DOI - 10.5752/P.1678-9563.2012v18n1p73. Psicologia em Revista, [S.I.], v. 18, n. 1, p. 73-89, Set. 2012. Disponível em: http://periodicos. pucminas.br/index.php/psicologiaemrevista/article/view/3844

MENEZES, N. T. DE.; SCHULZ, V. L.; PERES, R. S. Breast cancer diagnosis' psychological impact: a study since patients' reports in a support group. Estudos de Psicologia (Natal), v. 17, n. 2, p. 233-240, 2012. Disponível em: <http://dx.doi.org/10.1590/S1413-294X2012000200006>

PHILLIPS, K.; OSBORNE, R. H.; GILES, G. G.; GILLIAN, S. D.; APICELLA, C.; HOPPER, J. L.; MILNE, R. L. Psychosocial factors and survival of young women with breast cancer: a population-based prospective cohort study. Journal of Clinical Oncology, v. 26, n. 28, p. 4666-4671, 2008 . Disponível em: <http://ascopubs.org/doi/10.1200/JCO.2007.14.8718>

PRADO E. DO.; COSTA, J. R.; RAONE, D.; Presença e apoio: estratégias para ajudar a esposa a enfrentar o câncer de mama. Revista de Enfermagem UFPE. Recife, 10(11):3935-41, nov., 2016. Disponível em: <https://periodicos.ufpe.br/revistas/revistaenfermagem/article/view/11475>

RIIHIMÄKI, M.; THOMSEN, H.; BRANDT, A.; SUNDQUIST, J.; HEMMINKI, K. Death causes in breast cancer patients. Annals of oncology, v. 23, n. 3, p. 604-610, 2012. Disponível em: $<$ https://www.researchgate.net/publication/51140898_Death_causes_in_breast_canc er_patients>

SAWESI, S.; CARPENTER, J. S.; JONES, J. Reasons for non adherence to tamoxifen and aromatase inhibitors for thetreatment of breast cancer: a literature review. Clinical Journal of Oncology Nursing. Jun, 2014. Disponível em: < https://cjon.ons.org/cjon/18/3/reasons-nonadherence-tamoxifen-and-aromataseinhibitors-treatment-breast-cancer-literature>

SHAKERI, J.; GOLSHANI, S.; JALILIAN, E.; FARNIA, V.; NOORIPOUR, R.; ALIKHANI, M.; YAGHOOBI, K. Studying the Amount of Depression and its Role in Predicting the Quality of Life of Women withBreast Cancer. Asian Pacific Journal of Cancer Prevention, v. 17, n. 2, p. 643-646, 2016. Disponível em: < http://koreascience.or.kr/article/ArticleFullRecord.jsp?cn=POCPA9_2016_v17n2_643

SOUZA, B. F. DE.; PIRES, F. H.; DEWULF, N. L. S.; INOCENTI, A.; SILVA, A. E. B. C.; MIASSO, A.I. Pacientes em uso de quimioterápicos: depressão e adesão ao tratamento. Revista da escola de enfermagem da USP, São Paulo, v. 47, n. 1, p. 61-68, Fevereiro. 2013 Disponível em: $<$ https://www.revistas.usp.br/reeusp/article/view/52853/0> 
VAHDANINIA, M.; OMIDVARI, S.; MONTAZERI, A. What do predict anxiety and depression in breast cancer patients? A follow-up study. Social psychiatry and psychiatric epidemiology, v. 45, n. 3, p. 355-361, 2010. Disponível em: <https://link.springer.com/article/10.1007\%2Fs00127-009-0068-7>

WEI, D.; LIU, X.; CHEN, Y.; ZHOU, X.; HU, H. Effective ness of physical, psychological, social, and spiritual intervention in breast cancer survivors: An integrative review. AsiaPacific Journal of Oncology Nursing, v. 3, n. 3, p. 226, 2016. Disponível em: $<$ https://www.ncbi.nlm.nih.gov/pmc/articles/PMC5123516/> 